\title{
Remote assessment of physiological parameters by non-contact technologies to quantify and detect mental stress states
}

\author{
Frédéric Bousefsaf, Choubeila Maaoui, Alain Pruski \\ LCOMS - University of Lorraine \\ 7 rue Marconi, 57070 Metz, France \\ \{frederic.bousefsaf, choubeila.maaoui, alain.pruski\}@univ-lorraine.fr
}

\begin{abstract}
We present and investigate, in the first part of this paper, a set of published works that may be employed to remotely quantify mental stress based on physiological signals by non-contact means. Several techniques can be used to this purpose, thermal imaging being currently the most advanced in our knowledge. Webcams correspond to a ubiquitous and to the most accessible techniques in the particular purpose of mental stress detection. After all these theoretical reminders, we present a pilot study based on a new framework that was developed to detect mental workload changes using video frames obtained from a low-cost webcam. To induce stress, we have employed a computerized Stroop color word test on twelve subjects. The results offer further support for the applicability of mental workload detection by remote and low-cost means, providing an alternative to conventional contact techniques.
\end{abstract}

Keywords-mental stress; non-contact; human physiology

\section{INTRODUCTION}

Stress has repeatedly been associated with an increased risk for cardiovascular disease by primarily impacting blood pressure [1]. Stressful life events may result in acute and chronic depressive episodes where other physicals symptoms may appear: fatigue, weight loss, headaches and even digestive problems. Also, depression corresponds to a risk factor for coronary heart disease [2]. To reduce harmful effects caused by stress to the physical condition, biofeedback using relaxation and meditation exercises [3] [4] can be proposed to the patient. A proper food hygiene, sleep and a regular physical activity are factors that naturally decrease the impact of stress on the organism.

The association between affective states and computers has been popularized by R. Picard [5] who herein created the affective computing scientific domain. In these kinds of human-machine interactions, the computer is able to quantify affective states, stress and emotions [6] by using behavioral information and physiological parameters of the subject. Herein, stress detection and particularly mental workload changes are used to regulate the user-interface or the virtual environment to facilitate interactions [7]. Stress also impairs working memory and general cognitive function [4-5].
Quantifying stress by its physiological signature is a field of research that presents a particular and increasing interest, where physiological parameters like the heart rate and the electrodermal activity are reliable inputs to quantify different forms of stress [8-10]. However, contact sensors can be limited in some scopes of application where a specialist must install and monitor them [11]. In psychophysiological experiments, contact sensors may generate a negative bias by interfering with the user, resulting practically by an erroneous estimation [12].

The immediate objective of this paper is to review published works that may be included to the particular domain of stress detection based on physiological signals, sensed by non-contact means. Additionally, we present in the fifth section of this paper a pilot study to demonstrate that a simple and lowcost webcam can be employed to measure the instantaneous heart rate and quantify mental stress.

\section{STRESS DETECTION BY PHYSIOLOGICAL SIGNALS}

Physiological manifestations are orchestrated by the autonomic nervous system. The latter is split into two subbranches: the parasympathetic nervous system, which slows down the heart and reduces the size of the pupil (miosis) and the sympathetic nervous system, which in contrast accelerates the heart, dilates the pupil (mydriasis) and is responsible in sweating by the sweat (sudoriferous) glands. Stressors generally stimulate the sympathetic nervous system and inhibit the influence of the parasympathetic nervous system, these two components operating reciprocally.

\section{A. Standard physiological signals used for stress detection}

J.A. Healey and R. Picard [5] proposed to collect several physiological signals in order to determine relative stress level of drivers. Electrocardiogram, electromyogram, electrodermal activity and respiration were recorded during different driving conditions with contact sensors. A set of features were then extracted from raw signals to classify three levels of stress. They found that electrodermal activity and heart rate measurements were closely correlated with stress. 
Additionally, Zhai and Barreto [6] have employed contact skin temperature sensor and pupil diameter to separate stress states from calm states during computer work. To induce stress, they have used an interactive version of the Stroop color word test, a particular interference test. Shi and his colleagues [13] introduced in their work a method that considers the physiological signature of the user being observed. In fact, it is particularly difficult to propose a user-independent system because of the natural variations of physiology between each person. Electroencephalograms were employed to gather beta waves concurrently with other physiological signals [14]. Nevertheless, experiments are hard to conduct because of the invasiveness of such a system.

Only the cardiac activity can be used to detect stress [15] [4], specifically when computing the Heart Rate Variability (HRV), a factor that it is closely correlated to the autonomic nervous system. Time analysis of the HRV can be computed to observe the regularity of this series. Frequency analysis is performed to extract the power in particular frequency bands [16] using the Fast Fourier Transform to estimate the power spectral density. The acquisition is conventionally realized using contact ECG sensors but the cardiovascular pulse wave, assessed by photoplethysmography [17] can provide important information that are correlated with stress, especially the amplitudes of the pulse signal that reflects peripheral vasoconstriction or vasodilatation effects.

\section{B. Machine learning algorithm to quantify stress}

Different machine learning methods can be employed to quantify stress. $k$-nearest neighbors algorithm, Bayesian networks [6] support vector machines [13] natural neural networks or even linear discriminant analysis [9]. All the models need to be trained offline before being used in realtime.

\section{CONVENTIONAL VS NON-CONTACT TECHNIQUES}

In intensive care units, the monitoring of vital signs like the heart rate and the saturation of peripheral oxygen $\left(\mathrm{SpO}_{2}\right)$ are performed using contact pulse oximetry devices. These sensors are frequently plagued by motion artefacts [18] leading to frequent interventions of the medical staff due to false alerts [19]. When employing contact electrocardiographic (ECG) sensors, the presence of training personnel is required to place the electrodes onto the body of the patient. These precautions are necessary to avoid corrupted and noisy acquisitions. Noncontact technologies that are used to measure physiological signals are also sensitive to motion artifacts, except that movements are recordable when imaging devices are employed.

In some particular cases, like patients with burns, wounds or infections for example, conventional contact sensors may be inappropriate and even unusable. Concurrently, non-contact devices may be employed when allergic reactions to conductive gels are encountered. Some accessories and even some sensors must be replaced after each use for hygienic measures. Herein, non-contact devices are employed to reduce risks of infection and instrumentation coasts [19].

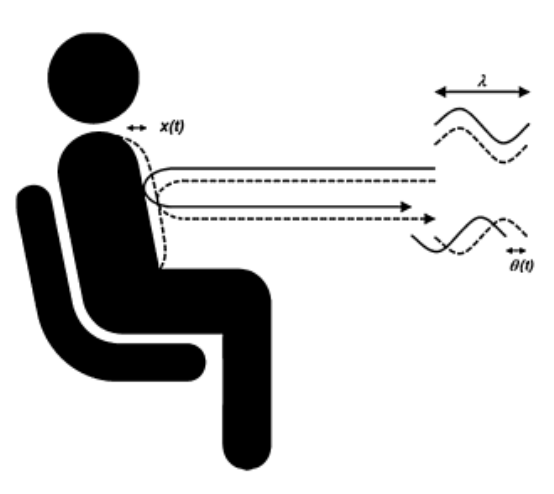

Fig. 1. Illustration of a Doppler effect on the chest. The frequency and the phase of the reflected signal are slightly different from those of the source signal. Figure extracted from [11].

The use of non-contact means to detect physiological signals is particularly advantageous in affective computing and psychophysiology, where stress or emotions are measured. In these psychophysiological experiments, contact sensors may generate a bias by interfering with the user, resulting practically by erroneous stress quantization or emotion misclassification [12]. Herein, non-contact technologies are non-invasive but not necessarily non-intrusive.

\section{MEASUREMENT USING NON-CONTACT TECHNOLOGIES}

Remote measurements of physiological signals are often accomplished using imagining devices. Thus, we will split methods based on digital cameras and webcams (visible spectrum) to the methods based on thermal imaging (infrared spectra). Concurrently, Doppler radars were used to extract heart and respiratory rates [11]. Recent works demonstrate that even speech contains hidden biological information [20] that can be assessed using microphones.

\section{A. Doppler}

Volumetric changes are orchestrated by the heart muscle (myocardium) contractions, which are partially transmitted to the chest. Radars based on the Doppler effect were proposed by E.F. Geneker [21] for sensing the heartbeat and the respiration remotely. These two physiological parameters are combined when observing chest movements, thus the challenge consists in efficiently separating them [22]. Lasers can be employed to measure the small displacements of the chest that cause frequency and phase changes (see Fig. 1) in the reflected signal [11].

Digital signal processing and adaptive filtering techniques are employed to properly extract and separate the cardiac signal from the respiration [22].

\section{B. Thermal imaging}

\section{1) Sensing of physiological signals}

The heart ejects a quantity of blood that synchronously travels through the arterial network before returning to the heart by the venous network. The propagation of the cardiac pulse generates modulations in the temperature of tissues, which are produced by convection and conduction. 


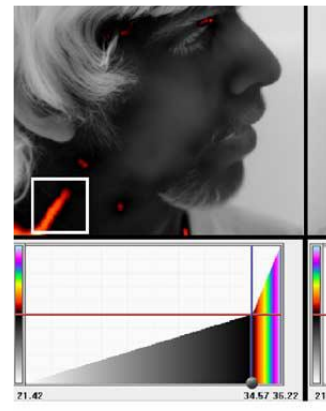

(a)
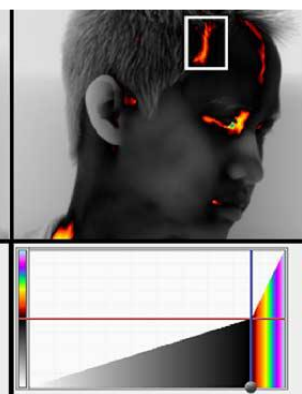

(b)
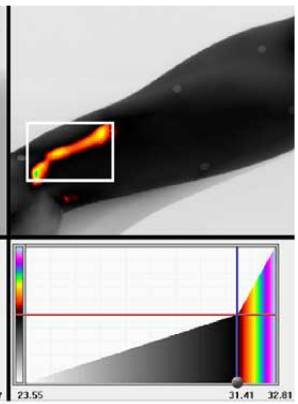

(c)

Fig. 2. The carotid (a) the temporal artery (b) and even the radial artery (c) are useful locations that can be employed to sense the pulse. Figure extracted from [23].

The skin temperature waveform reflects the cardiac pulse waveform, the pulse rate being perceptible using particular thermal cameras [23]. The effect is even more perceptible in superficial blood vessels, like the carotid for example (see Fig. 2).

The technology is completely passive (emits no energy) and one of the most challenging aspects when using thermal cameras is to automatically track the vessels and recover the pulse from composite signals using particular processing techniques [12]. Mid-wavelength infrared cameras [23] and long-wavelength infrared cameras [24] can be employed to recover the cardiovascular pulse wave and the respiration, by tracking temperature fluctuations around the nostrils area [25].

\section{2) Stress markers using thermal areas of interest}

Perspiration [26] can be detected by tracking changes in temperature on the maxillary area (Fig. 3). These fluctuations are modulated by the sympathetic nervous system and produce a response similar to the electrodermal activity, measured typically on the palm or fingers. Previous works of these authors [27] demonstrated that an increase of temperature in the supraorbital and periorbital areas, generated by an increased blood flow, corresponds to a ubiquitous manifestation of stress.

\section{Digital cameras and webcams}

Digital cameras and webcams were employed to detect and compute heart and breathing rates [28-30]. The principle, based on PhotoPlethysmoGraphy (PPG) consists in observing light variations on the skin to recover the cardiovascular pulse wave.

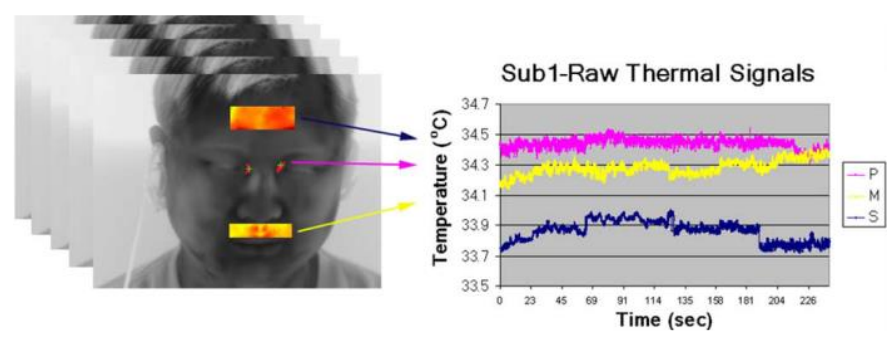

Fig. 3. The supraorbital, periorbital and maxillary areas present temperature changes that are correlated with stress. Figure extracted from [26].

This optical technique is mainly implemented in contact pulse oximetry sensors where infrared wavelengths are employed to detect the pulse wave. Considered in this case as noise, ambient light is now an illumination source used for PPG exploitation via high sensitivity cameras and webcams. The main drawback of this technique is that PPG signals are susceptible to motion-induced artifacts, particularly when dealing with webcams and ambient light.

Independent component analysis, a blind source separation method, has been proposed by Poh et al. [28] to remove noise artifacts from face imaging PPG signal. Sun et al. [29] have compared performances between a low-cost webcam and a high-sensitivity camera to assess HR and pulse rate variability. They conclude that the functional characteristics of a $30 \mathrm{fps}$ webcam are comparable to those of a 200 fps camera when interpolating signals to improve the time domain resolution. We have recently developed [30] a robust method to compute the HRV using the $u^{*}$ channel of the CIE $L^{*} u^{*} v^{*}$ color space combined to a skin detection, an essential step that improves signal to noise ratio (Fig. 4).

\section{Microphones (speech)}

People communicate basic linguistic information when they speak. The formants, the observation of relevant frequencies in the sound spectrum, indicate the phonetic quality of a vowel. It appears that the voice also contains important biological information. The cardiac activity causes short increments in the vowel speech formants [20]. This way, standard microphones can be employed to remotely detect and compute the instantaneous heart rate. Herein, noise artefacts are removed from the time-frequency representation of the raw signal. The main limitation of this method is that patients need to speak and keep a constant tone. Thus, patients with insufficient respiratory lung volume were not able to properly use the system [20].

\section{E. Capacitively coupled ECG}

Just like traditional ECG measurements, electric potentials are sensed using a couple of polarized electrodes. A conducting electrolyte gel is often used to ensure a proper resistive contact between the skin and the electrodes.

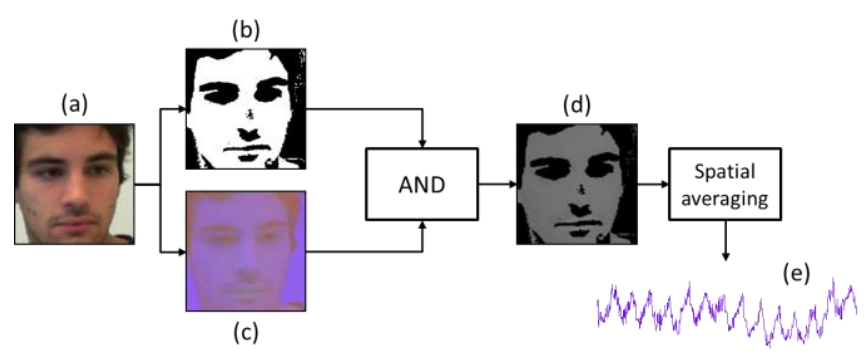

Fig. 4. Algorithm overview. The face is automatically detected on each frame (a). Pixels that contain PPG information are isolated by a skin detection (b). The RGB color space is converted to the CIE $L^{*} u^{*} v^{*}$ color space (c). The $u^{*}$ frame is merged with the skin detection mask by a combinational AND operation (d). A spatial averaging step is performed to transform a set of frames into a single raw signal (e). 

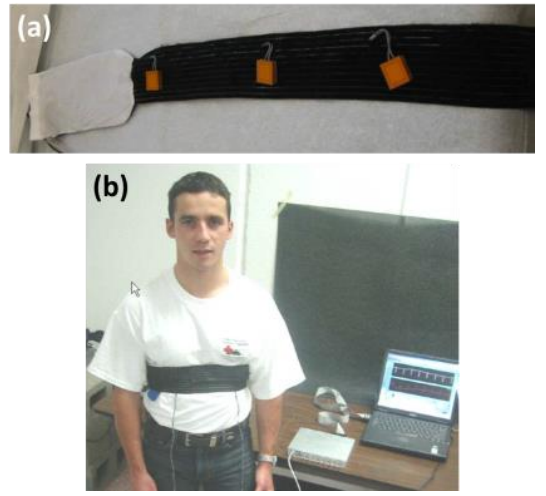

Fig. 5. Capacitive sensors can be attached to a strap (a). The belt can be placed around the chest and enables ECG monitoring without being in direct contact with skin (b). Figure extracted from [31].

Capacitive electrodes were developed to avoid this constraint and risks of skin irritation when monitoring ECG signals for long-term periods [31]. The system is able remotely sense these bioelectric signals but needs to operate in close proximity to the skin. These sensors [Fig. 5 (a)] need to be relatively sensitive to detect ECG signals and also robust to environment interferences. The system is not in direct contact with skin and can be placed over clothing [Fig. 5 (b)]. Recent work from Prance and his colleagues [32] demonstrates that electric-potential sensors can be used to measure the electric field at distances of up to $40 \mathrm{~cm}$ from the subject body.

\section{PILOT STUDY}

We have proposed [33] a new filtering technique that was developed to remotely and robustly recover the instantaneous heart rate signal concurrently to photoplethysmographic amplitudes fluctuations from video frames acquired by a lowcost webcam. Thus, a continuous wavelet transform filtering method was developed to precisely recover cardiac parameters of all participants.

Orchestrated by the autonomic nervous system, a peripheral vasoconstriction appears under stressful situations and leads PPG amplitudes to decrease [34]. We have employed these parameters to form a curve that represents mental workload changes for each of the 12 participants that were performing a computerized and interactive version of the Stroop [8] color word test (see Fig. 6).
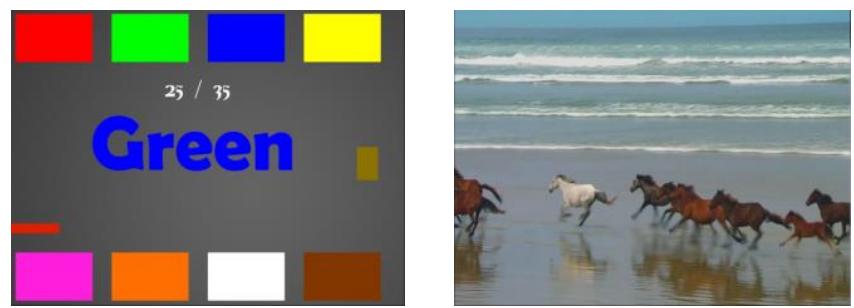

Fig. 6. Screenshots of the interactive application: during the Stroop color word test (left picture) and the first relaxation video (right picture) that starts right after the training session.
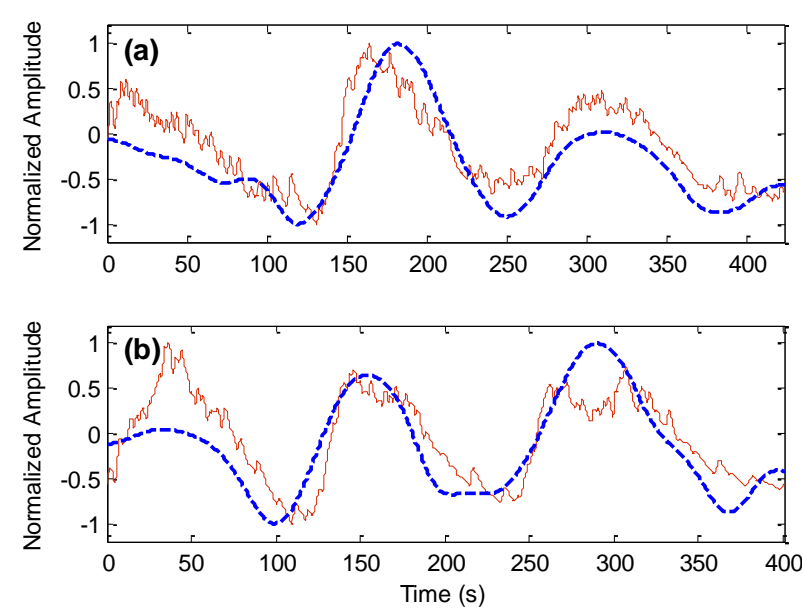

Fig. 7. Results of the mental workload detection for the participant \#11 (a) and \#12 (b). The dashed-line plot corresponds to the webcam-derived workload signal and the solid-line plot to the electrodermal trace.

Briefly, the participant has 3 seconds to click on the colored box that corresponds to the word printed in the center of the monitor (Fig. 6). Some words are printed in a color not denoted by the name (incongruent, e.g. the word "green" printed in a blue ink) while the others are printed on the right color (congruent, e.g. the word "yellow" written in yellow).The participants performed three sessions of the color word test, i.e. a one minute training session to familiarize the user with the virtual interface and two stress sessions. Each session are separated by a one minute relaxation session. A stressful music is played during both stress sessions and an alarm siren is launched the 10 last seconds.

Also, the electrodermal activity was recorded using a contact skin conductance sensor. This particular signal reflects sympathetic arousal and was compared to the mental workload curves assessed by the webcam (Fig. 7). Generally, a significant increase on mean and derivative values is perceptible between relaxation and stress sessions, independently of the measurement techniques. The boxplot of means printed in Fig. 8 gives an estimation of the mental workload curves computed with the webcam measurements. As for the electrodermal activity, these curves tend to decrease during relaxation sessions and, in contrast, tend to increase during stress sessions.
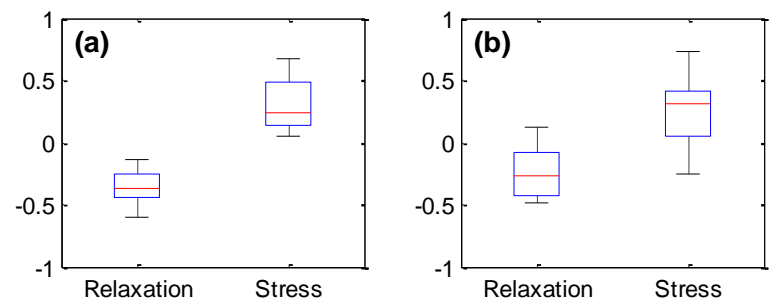

Fig. 8. Boxplots representing global average measurements of the webcam (a) and the electrodermal activity (b) for the three relax sessions and the two stress sessions. 


\section{CONCLUSION}

The results presented in this study [33] demonstrate the feasibility of using the cardiac response derived from a lowcost webcam to assess mental workload changes. The processing methods are motion-tolerant and robust to light deficiency [30]. The instantaneous heart rate can be properly assessed even in presence of strong motion artifacts.

Herein, we have demonstrated that webcams correspond to relevant non-contact sensors that can be employed to quantify the mental workload changes of a participant by computing a set of basic parameters extracted from the cardiac activity. Another challenging aspect is to integrate different modalities to recognize specific emotions. For example, body postures, facial expressions and gaze tracking can be extracted from input webcams frames. Even prosodic information can be sensed using built-in microphones. Other technologies, like thermal imaging are very promising in psychophysiology because they can sense particular temperature changes orchestrated by sympathetic arousal.

\section{REFERENCES}

[1] T.G.M. Vrijkotte, L.J.P. van Doornen, E.F.C. de Geus, "Effects of work stress on ambulatory blood pressure, heart rate, and heart rate variability," Hypertension, vol. 35, pp. 880-886, 2000.

[2] S.J. Bunker, D.M. Colquhoun, M.D. Esler, et al., "“'Stress” and coronary heart disease: psychosocial risk factors," The Medical Journal of Australia, vol. 178, pp. 272-276, 2003

[3] A. Goshvarpour, A. Goshvarpour, S. Rahati, "Analysis of lagged Poincaré plots in heart rate signals during meditation," Digital Sign. Proc., vol. 21, pp. 208-214, 2011.

[4] G.E. Prinsloo, H.G. Laurie Rauch, M.I. Lambert, et al., "The effect of short duration heart rate variability (HRV) biofeedback on cognitive performance during laboratory induced cognitive stress," Appl. Cognit. Psychol., vol. 25, pp. 792-801, 2010.

[5] J.A. Healey, R.W. Picard, "Detecting stress during real-world driving tasks using physiological sensors," IEEE Trans. Intelligent Transportation Systems, vol. 6, pp. 156-166, 2005.

[6] J. Zhai, A. Barreto, "Stress recognition using non-invasive technology," in Proc. 19th Int. Florida AI Res. Soc. Conf., Melbourne Beach, Florida, USA, May 2006, pp. 395-401.

[7] K. Hercegfi, "Heart rate variability monitoring during Human-Computer Interaction", Acta Polytechnica Hungarica, vol. 8, pp. 205-224, 2011

[8] A. H. Garde, B. Laursen, A. H. Jorgensen, B. R. Jensen, "Effects of mental and physical demands on heart rate variability during computer work,” Eur. J. Appl. Physiol., vol. 87, pp. 456-461, 2002.

[9] P. Melillo, M. Bracale, L. Pecchia, "Nonlinear heart rate variability features for real-life stress detection. Case study: students under stress due to university examination,” Biomed. Eng. Online, vol. 10:96, 2011.

[10] K. Yoshino, K. Matsuoka, "Personal adaptive methods to assess mental tension during daily life using heart rate variability," Methods Inf. Med., vol. 51, pp. 39-44, 2012.

[11] J. Kranjec, S. Begus, G. Gersak, J. Drnovsek, "Non-contact heart rate and heart rate variability measurements: A review," Biomedical Signal Processing and Control, vol. 13, pp. 102-112, 2014.

[12] I. Pavlidis, J. Dowdall, N. Sun, et al., "Interacting with human physiology," Computer Vision and Image Understanding, vol. 108, pp. 150-170, Oct. 2007

[13] Y. Shi, M.H. Nguyen, P. Blitz, et al., "Personalized stress detection from physiological measurements," in Int. Symposium on Quality of Life Technology, Las Vegas, NV, USA, June 2010
[14] M. Mauri, V. Magagnin, P. Cipresso, et al., "Psychophysiological signals associated with affective states," in Conf. Proc. IEE Eng. Med. Biol. Soc., Buenos Aires, Argentina, 2010, pp. 3563-3566.

[15] N. Hjortskov, D. Rissen, A.K. Blangsted, et al., "The effect of mental stress on heart rate variability and blood pressure during computer work,” Eur. J. Appl. Physiol., vol. 92, pp. 84-89, 2004.

[16] B. M. Appelhans, L. J. Luecken, "Heart rate variability as an index of regulated emotional responding," Review of General Psychology, vol. 10, pp. 229-240, 2006.

[17] J. Allen, "Photoplethysmography and its application in clinical physiological measurement," Physiol. Meas., vol. 28, pp. 1-39, 2007.

[18] X.F. Teng, Y.T. Zhang, "The effect of contacting force on photoplethysmographic signals," Physiol. Meas., vol. 25, pp. 13231335,2004

[19] L. Tarassenko, M. Villarroel, A. Guazzi, et al., "Non-contact videobased vital sign monitoring using ambient light and auto-regressive models," Physiol. Meas., vol. 35, pp. 807-831, 2014.

[20] A. Mesleh, D. Skopin, S. Baglikov, A. Quteishat, "Heart rate extraction from vowel speech signals," Journal of Computer Science and Technology, vol. 27, pp. 1243-1251, 2012 .

[21] E.F. Greneker, "Radar sensing of heartbeat and respiration at a distance with applications of the technology," in Radar 97 (Conf. Publ. No. 449), Edinburgh, UK, Oct. 1997, pp. 150-154.

[22] V. Vasu, C. Heneghan, T. Arumugam, and S. Sezer, "Signal processing methods for non-contact cardiac detection using Doppler radar," in IEEE Workshop on Signal Process. Syst., San Francisco, CA, Oct. 2010, pp. 368-373.

[23] M. Garbey, N. Sun, A. Merla, I. Pavlidis, "Contact-free measurement of cardiac pulse based on the analysis of thermal imagery," IEEE Trans. Biomed. Eng., vol. 54, pp. 1418-1426, Aug. 2007.

[24] Y. Chekmenev, H. Rara, A. Farag, "Non-contact, wavelet-based measurement of vital signs using thermal imaging," in Int. J. Graph. Vision Image Process., vol. 6, pp. 25-30, 2006.

[25] J. Fei, I. Pavlidis, "Thermistor at a distance: Unobtrusive measurement of breathing," IEEE Trans. Biomed. Eng., vol. 57, pp. 988-998, Apr. 2010.

[26] D. Shastri, A. Merla, P. Tsiamyrtzis, I. Pavlidis, "Imaging facial signs of neurophysiological responses,” IEEE Trans. Biomed. Eng., vol. 56, pp. 477-484, Feb. 2009.

[27] I. Pavlidis, J. Levine, "Thermal image analysis for polygraph testing," IEEE Eng. Med. Biol. Mag., vol. 21, pp. 56-64, Nov.-Dec. 2002.

[28] M.-Z. Poh, D. J. McDuff, R. W. Picard, "Advancements in noncontact, multiparameter physiological measurements using a webcam," IEEE Trans. Biomed. Eng., vol. 58, pp. 7-11, Jan. 2011.

[29] Y. Sun, S. Hu, V. Azorin-Peris, et al., "Noncontact imaging photoplethysmography to effectively access pulse rate variability," J. Biomed. Opt., vol. 18, 061205, Jun. 2013.

[30] F. Bousefsaf, C. Maaoui, A. Pruski, "Continuous wavelet filtering on webcam photoplethysmographic signals to remotely assess the instantaneous heart rate," Biomedical Signal Processing and Control, vol. 8, pp. 568-574, 2013

[31] J.M. Lee, F. Pearce, A. D. Hibbs, et al., "Evaluation of a CapacitivelyCoupled, Non-Contact (through Clothing) Electrode or ECG Monitoring and Life Signs Detection for the Objective Force Warfighter," RTO-MPHFM-109, pp. 25-1-25-10, 2004

[32] R.J. Prance, S.T. Beardsmore, P. Watson, et al., "Remote detection of human electrophysiological signals using electric potential sensors," Applied Physics Letters, vol. 93, 033906, 2008.

[33] F. Bousefsaf, C. Maaoui, A. Pruski, "Remote assessment of the heart rate variability to detect mental stress," 7th International Conference on Pervasive Computing Technologies for Healthcare (PervasiveHealth), Venice, Italy, May 2013, pp. 348-351.

[34] E. Peper, R. Harvey, I-M. Lin, et al., "Is there more to blood volume pulse than heart rate variability, respiratory sinus arrhythmia, and cardiorespiratory synchrony?,” Biofeedback, vol. 35, pp. 54-61, 2007. 